\title{
INFLUÊNCIA DO MÉTODO DE COLETA DE PARTÍCULAS PARA MEDIR A VAZÃO DE SÓLIDOS EM UM TRANSPORTADOR PNEUMÁTICO
}

\author{
V. M. B. MATSUMOTO ${ }^{1}$, T. F. PÁDUA \\ ${ }^{1}$ Universidade Federal de São Carlos, Departamento de Engenharia Química \\ E-mail para contato: viviane-matsumoto@hotmail.com
}

\begin{abstract}
RESUMO - O transportador pneumático de sólidos é importante devido à grande quantidade e diversidade de materiais que é capaz de transportar. Porém, os estudos em transportadores precisam vir acompanhados de medidas confiáveis de vazão de sólidos. O objetivo deste trabalho foi o de analisar a influência de dois métodos de amostragem sobre o valor da vazão mássica calculada para o transporte pneumático de partículas. Experimentos foram realizados introduzindo partículas esféricas de vidro (com $1 \mathrm{~mm}$ de diâmetro médio) em um transportador pneumático com a configuração de leito fluidizado circulante e foram usados dois galões distintos para recolher o material sólido para várias vazões de ar. Ambos tinham o mesmo tamanho e eram feitos do mesmo material, mas um possuía escape para o ar proveniente do transportador e o outro não. O reservatório com escape para o ar mostrou-se mais apropriado para amostragem da vazão de sólidos no caso do equipamento em questão, que não possuía escape de ar antes da caixa de amostragem de sólidos.
\end{abstract}

\section{INTRODUÇÃO}

Marcus et al. (1990) destacaram que a alternativa preferencial para transporte de material sólido em larga escala é o transporte pneumático, em que partículas são levadas por uma corrente de gás, comumente ar. Esses sistemas são importantes industrialmente, pois grande parte dos produtos e matérias primas se encontra no estado sólido (Mills, 2004; Marcus et al., 1990). Além disso, muitos sistemas de transporte pneumático podem ser utilizados no processamento de material sólido, se beneficiando da grande área de contato entre as partículas e a fase fluída, como ocorre na secagem em transportador (Rajan et al., 2008).

O objetivo deste trabalho foi a verificação da influência do método de amostragem sobre a vazão mássica medida no transportador. A mensuração adequada desta variável é fundamental para a caracterização do transporte pneumático, o que traz importância ao presente estudo. A medida da vazão de sólidos não é facilmente obtida nesses sistemas. Medidas indiretas são complexas e ainda pouco generalizadas (Mills, 2004), diferente do que ocorre com o escoamento de uma só fase (Delmée, 2003). A medida direta pode ser realizada em estudos no transportador pneumático através do desvio dos sólidos ao final do transportador (Patrocínio, 2006). 
Neste trabalho, a amostragem foi feita com o desvio dos sólidos por uma caixa de amostragem. Porém, avaliou-se se a possibilidade de escape de ar no recipiente de coleta de sólidos influenciava nas medidas de vazão. Para isto, foram usados dois galões distintos para recolher o material sólido desviado. Ambos tinham o mesmo tamanho e eram feitos do mesmo material, mas um possuía escape para o ar proveniente do transportador e o outro não. $\mathrm{O}$ transportador utilizado não possuía saída para o ar na região anterior à caixa de amostragem de sólidos para permitir o estudo do trecho descendente em toda a sua extensão. Assim, o transportador não incluía um ciclone e o ar saía pela secção superior do silo de armazenamento (onde havia uma malha para impedir o arraste de partículas). Neste caso, com os resultados apresentados, foi demonstrado que é indispensável utilizar escape para o ar no galão de coleta.

\section{MATERIAIS E MÉTODOS}

O transportador utilizado está esquematizado na Figura 1(a). O transportador estava em uma configuração proposta para aplicação em secagem (similar à de Lopes et al. 2011). Neste estudo utilizou-se o mesmo alimentador de sólidos que Pádua (2012), cuja função era evitar o desvio da corrente de ar para o conduto de alimentação (Mills, 2004).

Figura 1 - Transportador pneumático circulante utilizado $\mathrm{a}$ - transportador. $\mathrm{b}$ - caixa de amostragem (desenho esquemático).

(a)

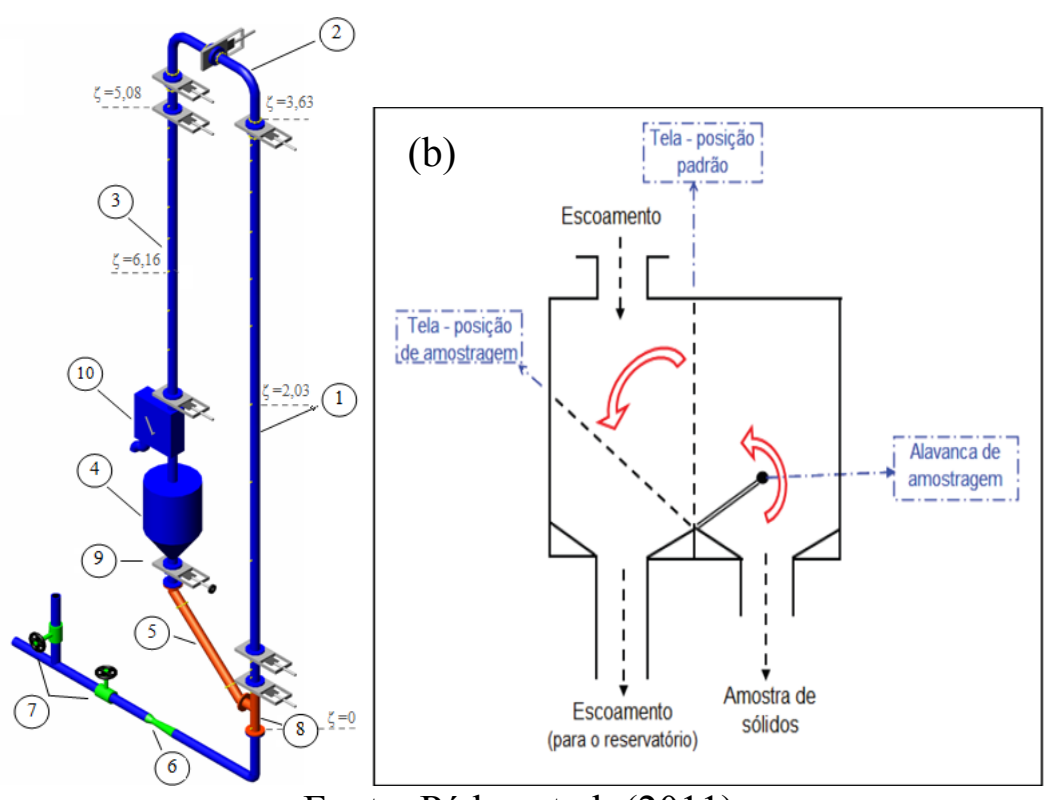

Fonte: Pádua et al. (2011)

Detalhes do aparato experimental e procedimentos podem ser encontrados em Lopes et al. (2011) e Patrocínio et al. (2006). Após completar o loop, as partículas chegavam a uma caixa de amostragem que poderia conduzi-las até um silo armazenador - onde o material sólido permanecia até ser reposto à corrente de transporte - ou poderia retirá-las do sistema, numa situação de amostragem. A vazão mássica foi obtida através da massa de partículas sólidas colhidas na amostragem e o tempo que se levou para que tal massa fosse retirada. A caixa de amostragem operava através de uma alavanca, como mostra a Figura 1(b). Os sólidos desviados eram levados por uma mangueira flexível até o reservatório de coleta (ou 
reservatório de amostragem) de particulado. Foram utilizados dois reservatórios distintos de coleta de amostras, apresentados na Figura 2, que se tratavam de galões vazios de água de 5L.

Enquanto a amostragem não era realizada, o escoamento levava ao reservatório de sólidos do transportador (silo de armazenamento, lado esquerdo da Figura 1.b), de formato cônico em sua base e com capacidade para armazenar cerca de $50 \mathrm{~kg}$ de sólidos. Uma válvula guilhotina na saída do silo servia como meio de controle para a vazão de sólidos.

Figura 2 - Reservatórios de amostragem. a - galão sem escape para o ar. b - galão com escape para o ar (reservatório de coleta com tela).

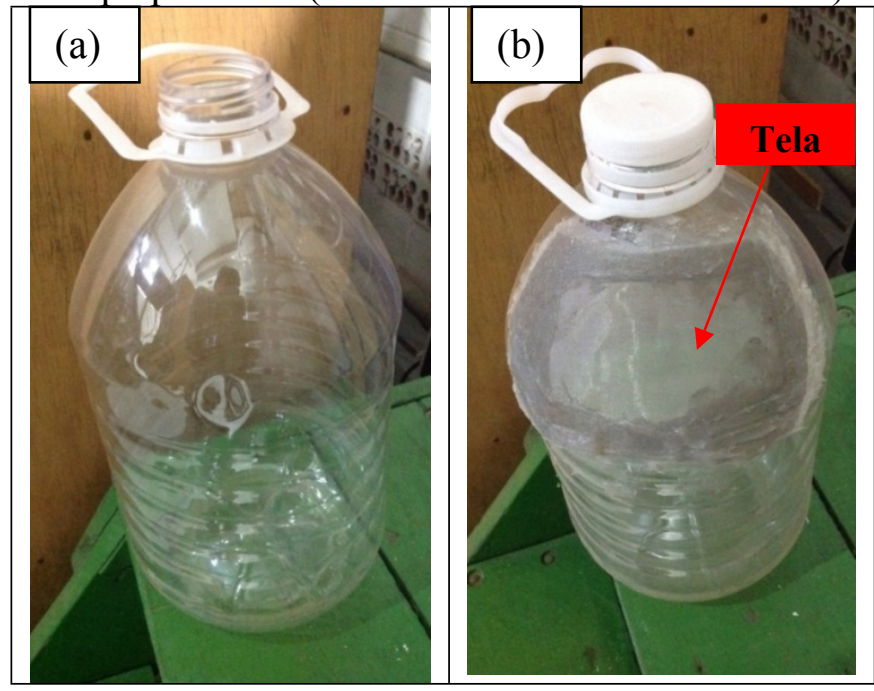

Fonte: Autor (2014)

As partículas retiradas durante a amostragem foram colhidas primeiramente por um galão (reservatório) todo fechado, mostrado na figura 2(a). Posteriormente, a amostragem foi feita utilizando-se um galão semelhante ao primeiro, que se distinguia apenas pela presença de um recorte em sua estrutura devidamente coberto por uma tela (reservatório com escape para o ar), mostrado na figura 2(b). O procedimento de amostragem foi seguido igualmente para os dois galões, de maneira que apenas a vazão volumétrica e o recipiente que colhia as amostras foram alterados durante o experimento.

O material sólido foi coletado de duas a três vezes em cada vazão volumétrica utilizada. As partículas eram constantemente repostas através da caixa de amostragem, de maneira a evitar grande alteração na altura do leito no silo. O tempo de amostragem variou $(10 \mathrm{~s}, 20 \mathrm{~s}$, $30 \mathrm{~s}, 40 \mathrm{~s}, 50 \mathrm{~s}$ e 60s) para que fosse possível avaliar os efeitos do tempo de coleta nos resultados para ambos os galões

\section{RESULTADOS E DISCUSSÕES}

\subsection{Resultados da amostragem com diferentes reservatórios}

A Figura 3 mostra as massas recolhidas em função do tempo de coleta da amostra para a vazãode $255,07 \mathrm{~m}^{3} / \mathrm{h}$.

Figura 3 - Massa recolhida em função do tempo de amostragem para a vazão volumétrica de $255,07 \mathrm{~m}^{3} / \mathrm{h}$. a - reservatório sem tela. $\mathrm{b}$ - reservatório com tela. 

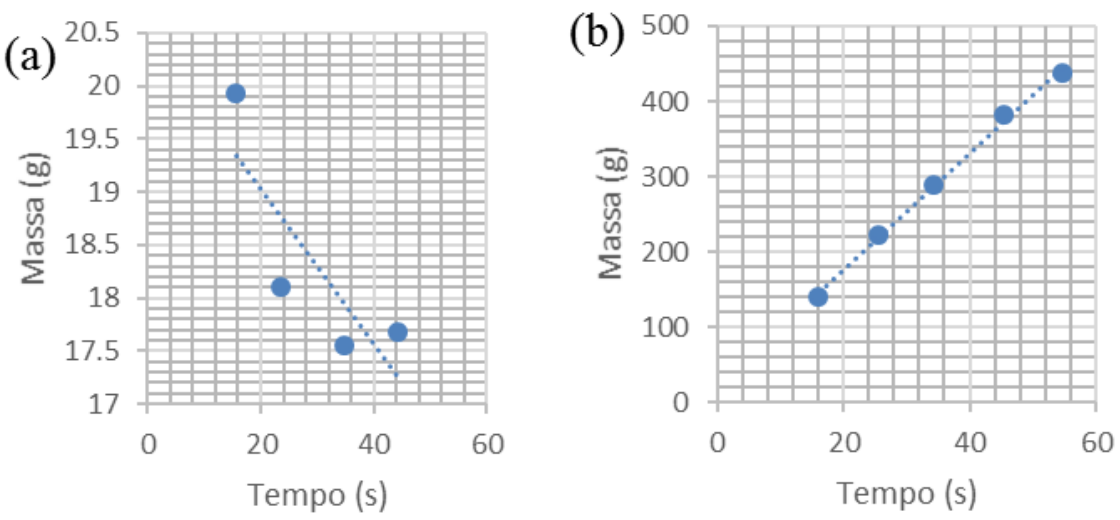

Fonte: Autor (2014)

A partir da Figura 3 percebe-se que a curva para o galão sem tela parece se estabilizar com o aumento do tempo de amostragem. Essa não é uma característica boa de amostragem, pois a massa coletada sempre deve aumentar com o tempo de amostragem. O comportamento linear obtido para o reservatório com escape para o ar é mais apropriado e tende a um valor de vazão mais estável em relação ao tempo de amostragem.

As discrepâncias entre os dois galões podem ser melhor observadas através dos gráficos de vazão mássica medida em função do tempo de coleta (Figura 4). Os resultados da Figura 4 indicam a maior reprodutibilidade dos ensaios com o galão com tela.

Figura 4 - Vazão mássica em função do tempo de amostragem para cada galão na vazão volumétrica igual a $255,07 \mathrm{~m}^{3} / \mathrm{h}$. a - reservatório sem tela. b-reservatório com tela.
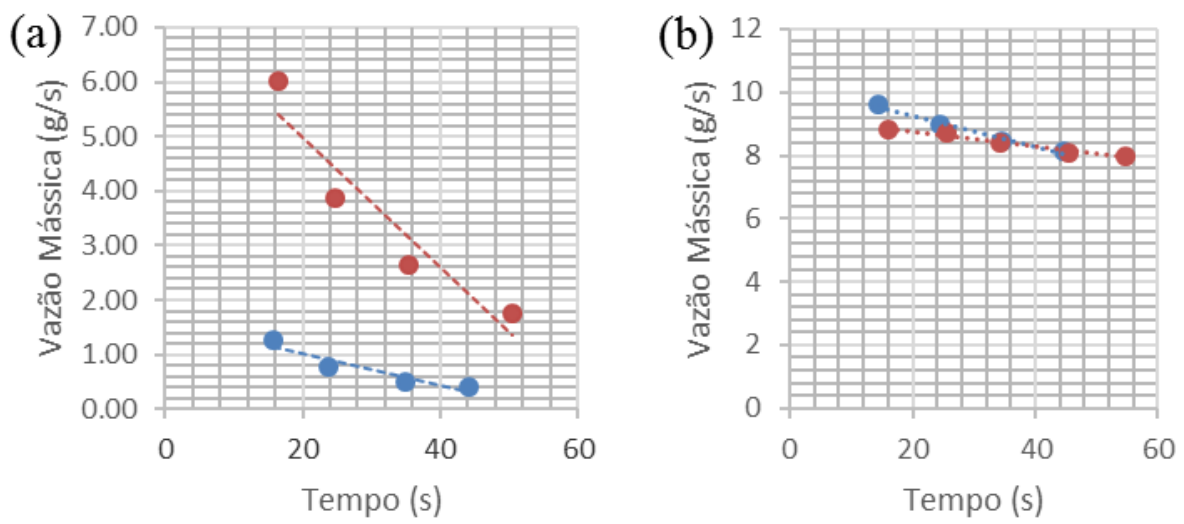

Fonte: Autor (2014)

Os resultados obtidos a partir do galão com tela aproximam-se dos resultados obtidos por Padua (2012) com o mesmo transportador e utilizando também um reservatório de coleta com escape para o ar, embora seu coeficiente de explicação $\left(\mathrm{R}^{2}\right)$ tenha sido mais satisfatório (0.989 contra 0.825). Ambos apresentaram uma curva crescente e a variação da vazão de sólidos está na casa dos 30\% quando se passa da menor vazão volumétrica para a maior, como pode ser observado na Figura 5. Idealmente, o método de medida não pode oferecer desvios sistemáticos e não houve evidências de que isso ocorreu no caso da caixa de amostragem usada (Figura 5). A diferença obtida nas retas ajustadas somente destaca a 
natureza do modelo empirico. Vale a pena também destacar que para o galão sem tela as vazões mássicas calculadas diminuíam conforme a vazão volumétrica aumentava, o que contraria a lógica de que elas deveriam ser diretamente proporcionais, bem como foi concluído por Pádua (2012). Já o galão com tela atende a tais expectativas (Figura 5).

O reservatório sem tela mostrou a inconsistência destacada na Figura 4, com relação à estabilização da massa coletada. Assim, é mais apropriado utilizar o reservatório com escape para o ar. Vale destacar que o tempo de amostragem influencia no valor calculado para a vazão, porém o reservatório com escape para o ar forneceu pouco mais de $3 \%$ de variação na medida com coleta de mais de 30 s de amostragem. Mais de 40 s forneceram variação em torno de $1,5 \%$. Nesse caso, pelo menos 30 s de coleta são recomendados.

Figura 5 - Vazão mássica de sólidos em função da velocidade superficial do ar para o galão com tela. Comparação com resultados de Pádua (2012). Barras de erro indicam intervalor de $95 \%$ de confiança.

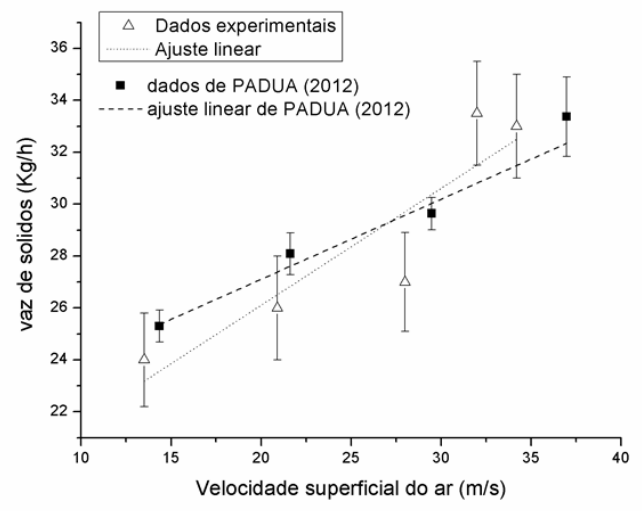

Fonte: Autor (2014).

\subsection{Explicação para os desvios obtidos com o reservatório sem escape}

A coleta de cada vez menos massa com a passagem do tempo de amostragem foi destacada a partir da Figura 3 para o galão sem tela. Esse comportamento é comum em vários níveis de vazão de ar utilizados. Isto pode ser observado experimentalmente ao se utilizar o reservatório sem escape para o ar, já que o galão era transparente. Quando a alavanca era girada para que a amostragem fosse feita, uma considerável quantidade de sólidos se depositava no galão fechado e, após alguns segundos, a passagem de sólidos praticamente cessava. Vale a pena destacar que não foi observada variação considerável da vazão de ar medida pelo dispositivo Venturi do trecho horizontal quando a alavanca era alterada para a posição de amostragem. Porém, a pressão anterior a caixa de amostragem era ampliada. Algumas hipóteses foram levantadas para explicar o comportamento inapropriado do reservatório fechado de coleta.

A melhor explicação encontrada é que o aumento da pressão no final do trecho descendente do transportador durante a amostragem pode promover um maior desvio da corrente de ar de transporte pelo conduto lateral, pois o ar tenderia a tomar esse caminho já que o silo de armazenamento encontrava-se em pressão atmosférica. O desvio da corrente de ar pode ter grande impacto na vazão alimentada de sólidos (Costa et al, 2004). Lopes et al. (2011) mostrou que esse desvio pode ser considerável até mesmo com a presença de bocais 


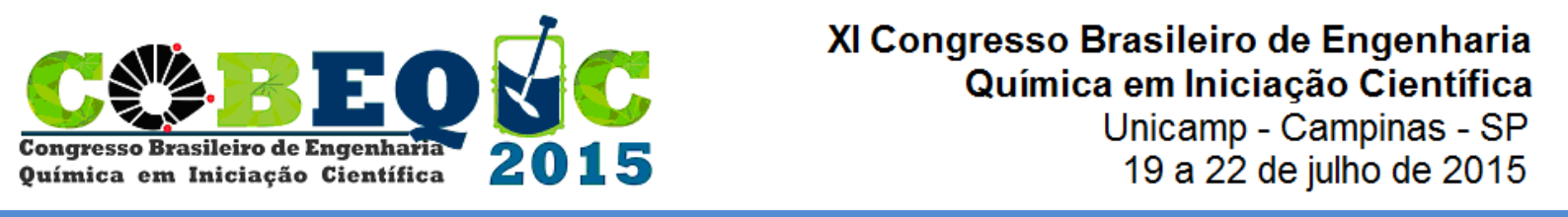

restritivos. O desvio do ar reduz ou até mesmo impede a corrente de sólidos de ser inserida no sistema (Costa et al, 2004; Lopes et al., 2011). A comprovação de que o reservatório fechado pode ampliar o desvio da corrente de ar de transporte pode ser testada ao se realizar medidas experimentais da perda de pressão no conduto lateral, que indicaria o desvio por ele (Costa et al, 2004). Ensaios serão realizados para isso e também para avaliar o impacto de ambas as amostragens nas pressões obtidas no trecho ascendente.

\section{CONCLUSÕES}

- Os galões com e sem escape de ar apresentaram resultados bastante discrepantes, o que comprova a influência dos meios de amostragem nas medidas;

- o galão sem tela deixa de coletar quantidades apreciáveis de sólido depois do início da amostragem, o que foi confirmado visualmente;

- os resultados servem de alerta para que se utilizem reservatórios de coleta com escape para o ar, ao menos em casos cuja amostragem seja realizada anterior à secção de escape de ar do transportador.

Hipóteses foram levantadas sobre as razões para o comportamento inapropriado da amostragem para reservatório fechado. Novos ensaios estão sendo realizados para comprovar essas hipóteses e avaliar melhor a reprodutibilidade dos métodos de amostragem.

\section{REFERENCIAS}

COSTA I. A.; CARMO M. F.; FREIRE J. T. Analysis of regime transition sand flow instabilities in a vertical conveying of coarse particles using different solids feeding systems. The Canadian Journal Of Chemical Engineering, v.82, p.48-59, 2004.

DELMÉE G. J. Manual de medição de vazão. Edgard Blucher, terceira edição, 2003.

LOPES C S. Estudo da alimentação de sólidos em sistemas de transporte pneumático. Tese de doutorado, programa de pós-graduação em engenharia química, UFSCar, São Carlos, 2011.

MARCUS, R. D.; LEUNG, L. S.; KLINZING, G. E.; RISK, F. "Pneumatic conveying of solids", Londres: Chapman and Hall, 575 p., 1990.

MILLS D. Pneumaticconveying design guide, Elsevier, segunda edição, 637 pag., 2004.

PÁDUA, T. F. Simulação de um alimentador Venturi em trecho ascendente no transporte pneumático de sólidos. Tese de doutorado, UFSCar, 2012.

PATROCÍNIO, A. B. Contribuição ao estudo do escoamento gás-sólido na presença de curvas. Tese de doutorado, programa de pós-graduação em engenharia química, UFSCar, São Carlos, 2006.

RAJAN, K. S.; DHASANDHAN, K.; SRIVASTAVA, S. N.; PITCHUMANI B. Studies on gas-solid heat transfer during pneumatic conveying. Int. J. Heat Mass Transfer, 51, 2801-2813, 2008.

\section{AGRADECIMENTOS}

Os autores agradecem à CAPES e ao CNPq pelo apoio financeiro. 